\title{
The burden of drug overdose on critical care units in East London, South Africa
}

To the Editor: Intentional self-harm accounted for $1.3 \%$ of non-natural deaths and $0.1 \%$ of all-cause mortality in South Africa (SA) in 2013. ${ }^{[1]}$ Fatal suicidal behaviour only represents a portion of the problem. The burden of non-fatal suicidal behaviour on our public health system is significant. Of particular concern is the downward trend in age of those affected, with an exponential increase in suicidal behaviour being seen among the younger generation. Overdoses account for $90 \%$ of non-fatal suicidal behavioural events among young people in SA. ${ }^{[2]}$

A small retrospective descriptive study was conducted at Frere Hospital in East London, Eastern Cape Province, to determine the recent burden of drug overdose on the high care unit (HCU) and intensive care unit (ICU) - jointly described as the critical care unit (CCU) - for the 1-year period from March 2014 to February 2015. 
The study only included adults and adolescents aged $\geq 13$ years. The patient data were collected from the admission registers in the CCU, as well as from individual patient folders. Casualty statistics were obtained from the casualty clerk's monthly data sheets.

The total number of overdose patients seen in casualty was 352 over the course of the year, with a mean of 29 patients per month. There were peaks noted in December (40 patients) and October (36 patients) - the same two months when male suicides peaked in SA in $2009 .{ }^{[3]}$ Overdose patients accounted for $6.8 \%$ of all patients seen by the physician on call in casualty throughout the year. Just over a fifth of overdose patients (71 patients) were admitted to the CCU, with $7.7 \%$ spending time admitted to the ICU.

There were 57 overdose patients admitted to the HCU during the year, with a mean of five patients per month. These patients accounted for $7.2 \%$ of the total number of HCU admissions, and $21.7 \%$ of all medical admissions to the HCU. There was a female predominance (61.4\%). Females were found to peak in the 13 - 19-year-old and 20 - 29-year-old age groups. Males peaked in the 20 - 29-year-old age group. Both sexes saw an early peak followed by a slow decline until the 60 - 69-year-old age group. The two most common categories of substances ingested were the tricyclic antidepressants (29.8\%) and the cholinesterase inhibitors or organophosphates (22.8\%). The median duration of stay in the HCU was 3 days, with the usual outcome (82.5\%) being transfer to a general medical ward. In total, the overdose admissions accounted for 190 days of admission in the HCU.

In the ICU, there were 28 overdose admissions in the year, with a mean of two patients per month. These patients accounted for $5.6 \%$ of all ICU admissions, and a quarter of all medical ICU admissions. There was no difference between the two genders in terms of number of admissions. Similar to the pattern noticed in the HCU, females peaked early in the 13 - 19-year-old age group, with males peaking slightly later in the 20 - 29-year-old age group. The two most common categories of substances ingested were the same as in the HCU (28.6\% tricyclic antidepressants, $25 \%$ organophosphates). The two main outcomes were transfer to the HCU or transfer to the general medical ward. The median duration of stay in the ICU was 3 days, with the overdose admissions accounting for 128 days of ICU admission in total. The cost implications are significant.
Comparison of these findings with those of a similar, larger study a 5-year (2006 - 2010) retrospective review conducted at Cecilia Makiwane Hospital (CMH), a nearby large referral hospital - reveals certain trends. ${ }^{[4]}$ Cholinesterase inhibitors were also one of the main substance categories resulting in CCU admission; however, they played a more predominant role at $\mathrm{CMH}$, accounting for $55 \%$ of all admissions. At both hospitals, females predominated; however, at Frere the difference was more pronounced ( $60 \%$ female, $40 \%$ male) than at $\mathrm{CMH}$ (53\% female, $47 \%$ male). The mean age (years) was similar at both hospitals: 30.86 at Frere (females 29.00, males 33.62) and 29.50 at $\mathrm{CMH}$ (females 27.91, males 31.31). The mean duration of stay in Frere's HCU was 3.33 days (79.92 hours), which was similar to CMH's CCU, where it was 3.31 days (79.32 hours). Overdose admissions peaked in the summer months at both hospitals (January at $\mathrm{CMH}$, December at Frere).

These findings demonstrate the recent trends and burden of overdoses on public hospitals in the Buffalo City Municipality, but they probably reflect a much larger, nationwide problem. These patients are managed by the Department of Internal Medicine at Frere Hospital in liaison with the Department of Psychiatry at CMH. There is no dedicated psychiatric unit at Frere Hospital, even though it is a tertiary referral centre. Improved availability and accessibility of psychiatric services as well as better co-ordination of medical and mental healthcare must become a public health priority.

\section{Kirsten Rowe}

Intern medical doctor, East London Hospital Complex, Eastern Cape, South Africa (currently Community Service Medical Officer, Galeshewe Day Hospital, Kimberley, Northern Cape, South Africa)

kirstenrowe@gmail.com

\footnotetext{
Statistics South Africa Mortality and causes of death in South Africa. Pretoria: Statistics South Africa, 2013:39.

2. Schlebush L. Suicidal behaviour. In: Van Niekerk A, Suffla S, Seedat M, eds. Crime, Violence and Injury in South Africa: 21 st Century Solutions for Child Safety. Cape Town: Psychological Society of South Africa, 2012:179-183

3. Matzopoulos R, Prinsloo M, Bradshaw D, et al. The Injury Mortality Survey: A National Study of Injury Mortality Levels and Causes in South Africa in 2009. Cape Town: South African Medical Research Council, 2013:29

4. Favara DM. The burden of deliberate self-harm on the critical care unit of a peri-urban referral hospital in the Eastern Cape: A 5-year review of 419 patients. S Afr Med J 2013;103(1):40-43. [http://dx.doi. org/10.7196/SAMJ.6231
}

S Afr J Med 2016:106(3):227-228. DOI:10.7196/SAMJ.2016.v106i3.9933

\section{Thank you, Janet Seggie}

Prof. Janet Seggie joined the Health and Medical Publishing Group (HMPG) in November 2012 as Editor-in-Chief of the South African Medical Journal (SAMJ), bringing with her the experience gained in an outstanding academic and clinical career that spanned the universities of the Witwatersrand and Cape Town.

Her impact on the journal has been significant, ensuring that it is a source of medicopolitical news, up-to-date comment and opinion, and excellent research that is highly relevant to our challenging medical environment in South Africa. She has also offered a publication platform for colleagues from other countries on the continent, who rarely have the chance to publish their research in difficult medical and academic environments.

Under Janet's tenure, the SAMJ once again took on an educational role by incorporating CME within its pages - a highly successful move that has attracted the contributions of some of our top medical academics.

We wish Janet well in her well-deserved retirement, and thank her sincerely for her contribution. 